\title{
Patients' preference for general or regional anaesthesia for caesarean deliveries at a district hospital, Free State, South Africa
}

\author{
J Hanekom, ${ }^{1} \mathrm{MB}$ ChB student; R Hattingh, ${ }^{1} \mathrm{MB}$ ChB student; W Nel, ${ }^{1} \mathrm{MB}$ ChB student; K Thirion, ${ }^{1} \mathrm{MB}$ ChB student; \\ C Willemse, ${ }^{1} \mathrm{MB}$ ChB student; G Joubert, ${ }^{2} \mathrm{BA}, \mathrm{MSc}$; C Boltman, ${ }^{1} \mathrm{MB}$ ChB, MFamMed, DA (SA); J Botes, ${ }^{1} \mathrm{BA}, \mathrm{MHPE}$
}

${ }^{1}$ Department of Family Medicine, Faculty of Health Sciences, University of the Free State, Bloemfontein, South Africa

${ }^{2}$ Department of Biostatistics, Faculty of Health Sciences, University of the Free State, Bloemfontein, South Africa

Corresponding author: C Boltman (boltmanc@ufs.ac.za)

\begin{abstract}
Background. Informing patients about available anaesthesia options allows patients to share in decision-making.
Objectives. To determine the preference for general or regional anaesthesia among patients admitted for elective caesarean delivery at a district hospital in Bloemfontein, South Africa, and whether the patients were informed about their anaesthesia options by their doctor. Methods. Consecutive sampling was used. Patients $\geq 18$ years old and who had had a caesarean delivery under general or regional anaesthesia participated in the study. Data were collected using a questionnaire, which was completed during formal interviews with patients.

Results. Of the 50 patients interviewed, $30(60.0 \%)$ preferred regional anaesthesia. With regard to the current surgery, $58.0 \%$ stated that they were informed of the available anaesthesia options.

Conclusion. The majority of the patients in our study preferred regional anaesthesia. Although only 58.0\% reported that they received information, many of those valued the doctor's opinion in their choice of anaesthesia.
\end{abstract}

S Afr J Obstet Gynaecol 2019;25(2):32-34. https://doi.org/10.7196/SAJOG.2019.v25i2.1444

Many patients have limited knowledge about their anaesthesia options for surgical procedures. ${ }^{[1]}$ Good doctor-patient communication is required prior to a procedure and patients should be informed of the advantages and disadvantages of each anaesthesia option. ${ }^{[1]}$ Involving patients in decision-making may decrease preoperative anxiety and improve patient satisfaction with the outcome. ${ }^{[1,2]}$

A Swiss study assessed patients' preference for participating in decision-making during their treatment, especially regarding anaesthesia options. ${ }^{[2]}$ Almost all of the 197 participants (94\%) expressed wanting to participate in decision-making and $62 \%$ indicated that they had received the desired level of shared decision-making. Owing to shared decision-making, $88 \%$ were highly satisfied and $12 \%$ were adequately satisfied with their treatment.

Patients treated at public health facilities in South Africa (SA) generally do not belong to a medical aid scheme and are of lower socioeconomic and educational status. ${ }^{[3]}$ In a Canadian study that compared patients' choices in context of socioeconomic and educational variables, researchers found that patients from communities with lower levels of education or lower income were more likely to refuse regional anaesthesia (epidural block) as pain management during labour. ${ }^{[4]}$ A study from Nigeria also found lower levels of education among participants who chose general anaesthesia over regional anaesthesia. ${ }^{[5]}$ In addition, the study showed age and religion to be significantly associated with the preference for general anaesthesia.

The aim of our study was to determine patients' preference for general or regional anaesthesia, what type of anaesthesia they received and what factors influenced their decisions.

\section{Methods}

Study design, setting, population and sampling strategy

This was an analytical cross-sectional study. Consecutive sampling was used. The target population was adult women admitted to a district hospital in Bloemfontein, SA, for an elective caesarean delivery. This hospital is a primary healthcare facility and receives patients from surrounding hospitals and clinics.

Patients who were 18 years or older, had undergone a caesarean delivery under general or regional anaesthesia, were willing to consent to an interview and indicated that they were able to communicate in English, were included. Only low-risk patients (i.e. who were not in a critical condition) were considered for inclusion.

\section{Data collection}

Data were obtained using an English questionnaire formulated from the objectives of the study. Questionnaires were presented to participants during formal personal interviews over a period of 5 weeks and completed by the interviewers according to the patients' responses. Interviews were conducted in the afternoons (between lunch and evening visiting hours) and at least six hours after surgery to minimise possible subjectivity due to the effects of anaesthesia.

\section{Pilot study}

The pilot study involved interviews with three patients using the structured questionnaire. Some questions were subsequently rephrased and additional response options were added. The results from the pilot study were not included in the main study. 


\section{Data analysis}

Data were analysed by the Department of Biostatistics, Faculty of Health Sciences at the University of the Free State (UFS). Results were expressed as frequencies and percentages.

\section{Ethical considerations}

The study was approved by the Ethics Committee of the Faculty of Health Sciences, UFS (ref. no: 21/2013). Permission to conduct the study was obtained from the chief executive officer of the hospital. Written informed consent was obtained from all participating patients and data were handled confidentially.

\section{Results}

A total of 50 patients who had had caesarean deliveries were interviewed. Most of the patients were between 21 and 40 years old $(n=40 ; 80.0 \%)$. Eight patients (16.0\%) were younger than 21 and two $(4.0 \%)$ were older than 40 . Three-quarters $(n=38 ; 76.0 \%)$ of the patients were black; the remainder $(n=12 ; 24.0 \%)$ represented other races. Only $26.0 \%$ of the patients $(n=13)$ reported previous surgeries, which mostly included a caesarean delivery $(84.6 \% ; n=11)$.

Results show that $58.0 \%$ of the patients were informed of their anaesthesia options before their procedure (Table 1). Almost all were aware of general (86.2\%) or regional anaesthesia (89.7\%). For the current procedure, 40 patients reported having had regional anaesthesia and 12 reported having had general anaesthesia; 3 patients reported that they had received both regional and general anaesthesia. Most patients (60.0\%) preferred regional anaesthesia. Of the 30 patients who noted factors that would influence their choice of anaesthesia, $56.7 \%$ said the doctor's opinion would

Table 1. Summary of the responses and perspectives of patients who received general or regional anaesthesia for a caesarean delivery

\begin{tabular}{ll}
\hline Questionnaire item & $\boldsymbol{n}(\%)$ \\
\hline $\begin{array}{l}\text { Have you been informed of the different types of } \\
\text { anaesthesia available for your surgical procedure? }(N=50)\end{array}$ & \\
$\quad$ Yes & $29(58.0)$ \\
$\quad$ Aware of general anaesthesia & $25(86.2)$ \\
$\quad$ Aware of regional anaesthesia & $26(89.7)$ \\
No & $21(42.0)$ \\
What type of anaesthesia did you have?* $(N=49)$ & \\
$\quad$ General & $12(24.5)$ \\
Regional & $40(81.6)$ \\
What type of anaesthesia would you prefer? $(N=50)$ & \\
$\quad$ General & $20(40.0)$ \\
$\quad$ Regional & $30(60.0)$ \\
When you chose the method of anaesthesia, what & \\
influenced your choice the most? $(N=30)$ & \\
Personal preference & $9(30.0)$ \\
$\quad$ Research & $3(33.3)$ \\
$\quad$ Fear & $5(55.6)$ \\
$\quad$ Opinion of friends/family members & $1(11.1)$ \\
Family members & $4(13.3)$ \\
Friends & $0(0)$ \\
Doctor's opinion & $17(56.7)$ \\
Would the type of procedure influence your preference for & \\
a certain type of anaesthesia? (N=49) & \\
Yes & $15(30.6)$ \\
No & $34(69.4)$ \\
&
\end{tabular}

influence their decision most, whereas $30.0 \%$ indicated personal preference as the deciding factor. Most of the patients (69.4\%) indicated that the type of procedure would not influence their preference for a specific type of anaesthesia.

\section{Discussion}

The majority of patients in our study preferred regional anaesthesia $(60.0 \%)$ during a caesarean delivery. In contrast, far fewer women opted for regional anaesthesia (29.9\%) in a study from Nigeria. ${ }^{[5]}$ In that study, the fear of feeling pain was stated as the most common reason for deciding against regional anaesthesia, whereas the most common reason for choosing regional anaesthesia was 'wanting to know what's going on' (78.9\%). General anaesthesia was chosen mostly owing to fear of being awake during the procedure $(71.9 \%) .^{[5]}$

In our study, $58.0 \%$ of patients stated that their doctor had informed them about the different types of anaesthesia available for their procedure. Patients may have forgotten that they had been informed or they may not have understood the options, despite telling the doctor otherwise. The observed uncertainty is similar to findings from a study conducted in Durban, SA, in which many patients remembered talking to an anaesthetist, but either did not understand or found the communication insufficient. ${ }^{[6]}$

From the reasons for selecting a specific type of anaesthesia, it is clear that many patients value their doctor's opinion. However, the results also indicate that fear contributes to patients' decisions. The attending doctor therefore has an important role in ensuring that patients are equipped with adequate, objective information to make an informed decision.

\section{Study limitations}

As convenience sampling over a limited period was used, the sample may not have been an accurate representation of Bloemfontein's patient population. After-effects of general anaesthesia may have influenced answers, as patients were interviewed only after surgery.

The questionnaire and interview were available only in English. On request, some questions were verbally translated to Afrikaans for some participants. However, there are many indigenous languages in SA and if a participant's understanding of Afrikaans or English was limited, it could have resulted in miscommunication.

Three patients indicated that they received both regional and general anaesthesia. This may well have been the case, as it is standard procedure to change to general anaesthesia if regional anaesthesia fails.

\section{Conclusion}

In this sample, $60.0 \%$ of the patients preferred regional anaesthesia for a caesarean delivery. Although only $58.0 \%$ remembered being informed about their options for anaesthesia, many of them indicated that they valued their doctor's opinion in deciding on the type of anaesthesia.

We hope to increase doctors' awareness of the importance of patient involvement in deciding on the type of anaesthesia prior to surgery, especially as joint decision-making helps to improve patient cooperation, reduces patient dissatisfaction after procedures and helps to give the patient peace of mind. Prior to elective surgery, doctors should evaluate what their patients already know about the available anaesthesia options and proceed to supplement their 


\section{CASE REPORT}

understanding or address misconceptions. Such consultation should include listening to a patient's preference and collaborating with the patient to decide on the best option.

1. Eyelade OR, Akinyemi JO, Adewole IF. Patients' perception and knowledge of anaesthesia and Eyelade OR, Akinyemi JO, Adewole IF. Patients' perception and knowledge of anaesthesia and
anaesthetists - a questionnaire survey. S Afr J Anaesth Analg 2010;16(4):28-31. https://doi.org/10 anaesthetists - a questionnaire

2. Flierler WJ, Nübling M, Kasper J, Heidegger T. Implementation of shared decision making in anaesthesia and its influence on patient satisfaction. Anaesthesia 2013;68(7):713-722. https://do org/10.1111/anae. 1219

3. Omotoso K, Koch SF. South African trends in medical aid coverage and stated healthcareseeking preferences: 2004-14. Dev South Afr 2017;34(5):575-592. https://doi.org/10.1080/03768 35X.2017.1360175
4. Liu N, Wen SW, Manual DG, Katherine W, Bottomley J, Walker MC. Social disparity and the use of intrapartum epidural analgesia in a publicly funded health care system. Am J Obstet Gynecol 2010;202(3):273.el-e8. https://doi.org/10.1016/j.ajog.2009.10.871

5. Bukar M, Kwari DY, Moruppa JY, Ndonya DN. Anaesthesia for caesarean delivery: Choice of technique among antenatal attendees in north-eastern Nigeria. J Obstet Gynaecol 2010;30(8):822 825. https://doi.org/10.3109/01443615.2010.513459

6. Naidu S, Gopalan PD. The informed consent process for anaesthesia: Perspectives of elective surgical patients at Inkosi Albert Luthuli Central Hospital, Durban, South Africa. S Afr J Anaesth Analg 2016;22(2):60-64. https://doi.org/10.1080/22201181.2016.1157963

Accepted 7 October 2019 